\title{
A WELL POSED PROBLEM FOR THE BACKWARD HEAT EQUATION
}

\author{
W. L. MIRANKER
}

1. Introduction. A boundary value problem is well posed in the sense of Hadamard if three conditions are met. These are existence, uniqueness, and continuous dependence of the solution on the boundary data. In recent years some attention has been given to improperly posed problems $[1 ; 2 ; 3]$. The point of view in these matters is directed at approximating in some stable way the solution of a problem which violates the last condition of well-posedness just described.

In this paper we will consider the backward heat equation, which leads to a classical improperly posed problem. To see that this is the case, consider the forward heat equation

$$
u_{t}=u_{x x}
$$

$$
t>0 \text {, }
$$

with the initial condition

$$
u(x, 0)=f(x)=n \sin n x .
$$

The solution is

$$
u(x, t)=n e^{-n^{2} t} \sin n x .
$$

Thus the forward heat equation has a sequence of solutions which tend to zero while their corresponding initial values tend to infinity. Thus the backward heat equation which consists of solving (1.1) for $t<T$ say, with data given for $t=T$ has solutions which do not depend continuously on their initial data.

The question of existence of a solution to the backward heat equation is also a delicate matter. To see this we observe that the solution of (1.1) and (1.2) may be written as

$$
u(x, t)=\int_{-\infty}^{\infty} f(\sigma) K(x-\sigma, t) d \sigma \equiv K_{T f}
$$

where

$$
K(x, t)=\frac{1}{2(\pi t)^{1 / 2}} \exp \left(-x^{2} / 4 t\right) .
$$

Therefore when a solution of the forward heat equation exists it is analytic for all $t>0$. Therefore one can only prescribe certain analytic

Presented to the Society, August 30, 1960; received by the editors April 1, 1960. 
data for the starting values of the backward heat equation. Moreover, since $f(\sigma)$ need not be analytic, the time over which the solution to the backward heat equation exists depends on the initial values.

In this paper we will consider the backward heat equation in $L^{2}$. We will show that in a subspace of $L^{2}$ consisting of functions whose Fourier Transforms have compact support, the backward heat equation leads to a well posed problem in the sense of Hadamard. We also provide a stable and convergent iteration scheme by means of which the solution may be computed.

2. Statement of results. Let $f(x) \in L^{2}(-\infty, \infty)$, i.e., $\|f\|^{2}=\int|f(x)|^{2} d x$ $<\infty, 1$ and let the Fourier Transform of $f(x)$ be denoted by

$$
F(\omega)=S f(x)=\frac{1}{(2 \pi)^{1 / 2}} \int e^{-i \omega x} f(x) d x .
$$

Let $\Omega$ be a fixed positive number and denote by $B$ the following subspace of $L^{2}$ :

$$
B=\left\{f(x) \in L^{2}|F(\omega) \equiv 0,| \omega \mid>\Omega\right\} .{ }^{2}
$$

For convenience we introduce the following:

Definition. A function $u(x)$ is said to have diffused from $f(x)$ in time $T$ if

$$
u(x)=u(x, T)
$$

with $u(x, T)$ given by (1.4).

Our result is given in the following:

THEOREM. Let $u(x) \in B$. Then to each $T>0$ there exists one and only one $f(x) \in B$ such that $u(x)$ has diffused from $f(x)$ in time $T . f(x)$ is given by

$$
\lim _{n \rightarrow \infty}\left\|f_{n}-f\right\|=0
$$

where

$$
\begin{aligned}
f_{n+1}(x) & =u(x)+\left(I-K_{T}\right)_{j}(x), \\
f_{0}(x) & =0 .
\end{aligned}
$$

Moreover, if $u(x)$ is changed to $u(x)+\Delta u(x)$ then the change $\Delta f$ in $f$ satisfies the relation

1 All integrals are taken from $(-\infty, \infty)$ unless otherwise specified.

2 The linearity of $S$ implies that $B$ is a linear manifold. That it is closed follows from the Plancherel theorem since $\int\left|f_{n}-f\right|^{2} d x=\int\left|F_{n}-F\right|^{2} d \omega$ assures us that $F_{n} \rightarrow F$ whenever $f_{n} \rightarrow f$. 


$$
\|\Delta f\| \leqq C\|\Delta u\|
$$

where $C$ is a constant.

Proof. Since

$$
S K_{r f}=(2 \pi)^{-1 / 2} e^{-T \omega^{2}} F(\omega),
$$

and $F_{0}(\omega)=0$, it follows from (2.3) that $f_{n} \in B$ for all $n$. Now making use of the fact that $S$ is of an isometry of $L^{2}$ onto itself, we have from (2.3),

$$
\begin{aligned}
\left\|f_{n+1}-f_{n}\right\| & =\left\|\left(I-K_{T}\right)\left(f_{n}-f_{n-1}\right)\right\| \\
& =\left\|\left(1-\frac{1}{(2 \pi)^{1 / 2}} e^{-T \omega^{2}}\right)\left(F_{n}-F_{n-1}\right)\right\| \\
& =\left[\int_{-\Omega}^{\Omega}\left|\left(1-\frac{1}{(2 \pi)^{1 / 2}} e^{-T \omega^{2}}\right)\left(F_{n}-F_{n-1}\right)\right|^{2} d \omega\right]^{1 / 2} \\
& \leqq\left(1-\frac{1}{(2 \pi)^{1 / 2}} e^{-T \Omega^{2}}\right)\left\|F_{n}-F_{n-1}\right\| \\
& =\theta\left\|f_{n}-f_{n-1}\right\| .
\end{aligned}
$$

Since $\theta<1$, the sequence $f_{n}(x)$ is a Cauchy sequence of functions in $B$. Since $B$ is closed, this sequence has a limit $f \in B$. (2.6) shows that $\left(I-K_{T}\right)$ is a continuous operator in $B$. Thus taking the limit as $n \rightarrow \infty$ in (2.3) we obtain

$$
u(x)=K_{T} f(x) .
$$

If $u(x)$ is replaced by $u(x)+\Delta u$, it follows from

$$
\begin{aligned}
f & =\lim _{N \rightarrow \infty} \sum_{n=0}^{N}\left(f_{n+1}-f_{n}\right) \\
& =\lim _{N \rightarrow \infty} \sum_{n=1}^{N}\left(I-K_{T}\right)\left(f_{n}-f_{n-1}\right)+f_{1} \\
& =\lim _{N \rightarrow \infty} \sum_{n=1}^{N}\left(I-K_{T}\right)^{n}\left(f_{1}-f_{0}\right)+f_{1} \\
& =\lim _{N \rightarrow \infty} \sum_{n=0}^{N}\left(I-K_{T}\right)^{n} u
\end{aligned}
$$

that 


$$
\Delta f=\lim _{N \rightarrow \infty} \sum_{n=0}^{N}\left(I-K_{T}\right)^{n} \Delta u .
$$

Thus

$$
\|\Delta f\| \leqq \frac{1}{1-\theta}\|\Delta u\| .
$$

This also proves the uniqueness of $f$. Q.E.D.

REMARK. The theorem assures both the convergence of $f_{n}$ to $f$ and the boundedness of $\Delta f$ in the $L^{2}$ sense. To satisfy ourselves that the instabilities of the backward diffusion process have not been masked by an integral mollifying process, we remark that the convergence of $f_{n}$ to $f$ and the boundedness of $\Delta f$ are assured also in the maximum norm. This we state as the following:

COROLlaRY. (a) The sequence $f_{n}$ produced in the previous theorem converges in the maximum norm, i.e.,

$$
\lim _{n \rightarrow \infty} \max _{x}\left|f_{n}(x)-f(x)\right|=0 .
$$

(b) The error $\Delta f$ defined in the previous theorem is bounded in the maximum norm, i.e.,

$$
\max _{x}|\Delta f| \leqq C\|\Delta u\| .
$$

Proof. The proof consists of the observation that in $B, L^{2}$-convergence implies $L^{\infty}$-convergence. Viz., if $f(x) \in B$ then

$$
\begin{aligned}
\max _{x}|f(x)| & =\max _{x} \frac{1}{(2 \pi)^{1 / 2}}\left|\int e^{i \omega x} F(\omega) d \omega\right| \\
& \leqq \max _{x} \frac{1}{(2 \pi)^{1 / 2}}\left(\int_{-\Omega}^{\Omega} d \omega\right)^{1 / 2}\left(\int_{-\Omega}^{\Omega} F^{2} d \omega\right)^{1 / 2} \\
& \leqq\left(\frac{\Omega}{\pi}\right)^{1 / 2}\|f\| .
\end{aligned}
$$

REMARK. (2.12) shows that the iteration scheme is stable even if arbitrarily large errors in the maximum sense are made in $u$, provided only that the corresponding $L^{2}$ norm of these errors is small.

REMARK. If the iteration scheme is implemented, numerically say, there is always the danger that a specific iterate will not be in $B$ because of errors in the actual means of implementation. However, it is clear that even though the successive functions so obtained do not 
appear to converge, the projections of the Fourier Transforms of this divergent series onto $|\omega|<\Omega$ are converging both in the $L^{2}$ norm and the maximum norm.

\title{
BIBLIOGRAPHY
}

1. J. Douglas, Jr. and T. M. Gallie, An approximate solution of an improper boundary value problem, Duke Math. J. vol. 26 (1959) pp. 339-347.

2. F. John, Numerical solution of the equation of heat conduction for preceding times, Ann. Mat. Pura Appl. ser. IV vol. 40 (1955) pp. 129-142.

3. C. Pucci, Sui problemi di Cauchy non "ben posti," Atti Accad. Naz. Lincei. Rend. Cl. Sci. Fis. Mat. Nat. vol. 18 (1955) pp. 473-477.

International Business Machines Corporation

\section{PERRON-FROBENIUS THEORY AND THE ZEROS OF POLYNOMIALS}

\author{
HERBERT S. WILF ${ }^{1}$
}

1. Introduction. Our purpose here is to show that many of the classical root location theorems for polynomial equations, normally derived by the methods of complex analysis, can be obtained easily, and in a purely algebraic manner, from the Perron-Frobenius theorems on matrices with nonnegative elements. An important result of this approach is a "minimax" principle which gives precisely the largest root of an equation which dominates the given one. It will be seen that from this principle the above-mentioned location theorems follow, and can be sharpened almost at will. Finally some applications to the theory of orthogonal polynomials will be made, the result again being a minimax principle for the largest zero from which twosided bounds can be deduced by specialization.

2. Cauchy's Theorem. Let $C$ be an $n \times n$ complex matrix, and let $C^{+}$be given by

$$
\left(C^{+}\right)_{i j}=\left|C_{i j}\right|, \quad(i, j=1, \cdots, n) .
$$

A lemma of Wielandt [1] asserts that if $C^{+}$is irreducible, $\gamma$ is any eigenvalue of $C$, and $r$ is the largest real eigenvalue of $C^{+}$, then $|\gamma| \leqq r$.

Let

Presented to the Society, January 24, 1961 ; received by the editors May 13, 1960.

1 This work was supported in part by the National Science Foundation. 